\title{
Relation between maternal lipid profile and pregnancy complications and perinatal outcomes.
}

\author{
Mohammed Abdu Helmy, ${ }^{1}$ M.B.B.Ch; Emad Maarouf Abd El-Latif, ${ }^{1}$ MD; Mofeed Fawzy Mohamed, \\ ${ }^{1} \mathrm{MD}$; Ahmed Fathy Abd-Elaziz, ${ }^{1} \mathrm{MD}$.
}

* Corresponding Author:

Mohammed Abdu Helmy

m.abdu@msn.com

\author{
Received for publication \\ November 3, 2020; Accepted \\ January 4, 2021; Published \\ online January 4, 2021.
}

Copyright 2020 The Authors published by Al-Azhar University, Faculty of Medicine, Cairo, Egypt. All rights reserved. This an open-access article distributed under the legal terms. where it is permissible to download and share the work provided it is properly cited. The work cannot be changed in any way or used commercially.

doi: 10.21608/aimj.2021.46596.1337.

${ }^{I}$ Department of obstetrics and Gynecology, Faculty of medicine, AlAzhar University (Cairo), Egypt.

\begin{abstract}
Background: Pregnant experiences physiological changes in maternal lipid metabolism to support fetal growth and development. In some cases a maladaptation occurs and exceeds the physiological range and dyslipidemia is recognized, some pregnancies pacing without associated pregnancy alterations and in pregnancies pacing with pathologies.

Aim of the study: determine the relationship between maternal lipid profile and pregnancy and perinatal complications. Besides, determine the cut - of value of each lipid profile components for predicting maternal compilations.

Patient and Methods: 164 pregnant who attended the Obstetrics and Gynecology department in Suez Canal Authority Hospital in Ismailia city, throughout the period May 2018 - October 2019. Pregnant were assessed clinically, obstetrically, and tested for lipid profile during 2nd and 3rd Trimester, for detecting any maternal or neonatal complications.

Results: 28 pregnant developed maternal complications [GHTN (3.66\%), Preeclampsia (2.44\%), GDM (3.05\%), IHCP (1.83\%), PTL (4.27\%), PTB $(3.05 \%)$ and ROM $(4.78 \%)$ ]. Lipid profile in complicated cases during 2nd/3rd trimester for TC, TG, LDL, and HDL were $189.3 \pm 4.8 / 243.2 \pm$ $4.8 \mathrm{mg} / \mathrm{dl}, 271.0 \pm 8.4 / 251.2 \pm 8.4 \mathrm{mg} / \mathrm{dl}, 110.8 \pm 5.6 / 114.2 \pm 5.6 \mathrm{mg} / \mathrm{dl})$ and $60.4 \pm 1.8 / 61.2 \pm 1.9 \mathrm{mg} / \mathrm{dl})$. We observed every $\mathrm{mg} / \mathrm{dl}$ elevation in maternal 3rd-trimester TG concentration was associated with an increased risk of GDM, GHTN, preeclampsia, and IHCP. Every $\mathrm{mg} / \mathrm{dl}$ increase in 3rd-trimester TG concentration was associated with an increased risk for SGA, LGA, and macrosomia.

Conclusion: maternal dyslipidemia is a risk factor and associated with the development and occurrence of maternal complications during pregnancy and affects neonatal outcomes.
\end{abstract}

Keywords: dyslipidemia; gestational hypertension; gestational diabetes; cholestasis; macrosomia.

Disclosure: The authors have no financial interest to declare in relation to the content of this article. The Article Processing Charge was paid for by the authors.

Authorship: All authors have a substantial contribution to the article.

\section{INTRODUCTION}

Pregnant experiences physiological changes in maternal lipid metabolism to support fetal growth and development. ${ }^{1}$ Maternal lipid metabolism varied during pregnancy and this condition was characterized by being progressive, increased with weeks of gestation. $^{2}$ There is adipose tissue expansion, and hepatic lipid synthesis increases and this physiological adaptation is associated with changes in Lipid profile during pregnancy. ${ }^{3}$

During normal pregnancy, there are increases in the blood concentration of TC, TG, LDL-C, and decreases in HDL-C. ${ }^{4}$ Accumulation of lipids in maternal tissues and the development of maternal hyperlipidemia occur. In some cases, a maladaptation occurs and these levels increase over a physiological range, and dyslipidemia is recognized. This condition occurs in some pregnancies pacing without associated pregnancy alterations and in pregnancies pacing with pathologies as preeclampsia (PE) and GDM. ${ }^{5}$

Our goal was to find if there is a relationship between maternal lipid profile and pregnancy complications and perinatal outcomes.

\section{PATIENT AND METHODS}

A Prospective Observational Study was conducted on 164 pregnant who attended regular prenatal health care visits in the obstetrics clinic in Suez Canal Authority Hospital in Ismailia city, during the period May 2018 - October 2019. 
Singleton pregnancy with gestational weeks $21-37$ of $18-40$ years old pregnant who naturally conceived was participated in this study. Pregnant with multiple pregnancies, had metabolic diseases or inherited diseases before pregnancy, Infection during early pregnancy, or conceived with ART were excluded.

All participated were requested to complete an extensive medical history and physical examination. Then, each Pregnant was assessed clinically, obstetrically, and tested for lipid profile during the period 21-24 weeks and repeated during the period 33-37 weeks.

All participants were antenatally followed-up from recruitment to delivery. Her medical status and occurrence of complication with pregnancy were collected from medical records .

In the present study, we tested an association between maternal lipid profile status of the pregnant mother and the possibility of complications for her or her fetus, or the occurrence of problems during or after childbirth. For maternal complications, GDM, GHTN, preeclampsia, ICP, PTB, and ROM were selected. For fetal conditions, SGA, LGA, and macrosomia were recorded. Regarding maternal outcome [delivery mode, Obstetric trauma (as Perineal injury (2nd -4th degree) or cervical injury], Obstructed labor and shifted to perfume CS delivery and occurrence of PPH. Information of newborn sex, birth weight, Apgar scores, and perinatal outcomes were recorded upon delivery .

Venous blood samples were taken from participants after overnight fasting. The blood samples were collected in a $3.5 \mathrm{ml}$ plan tube for serum preparation. One $\mathrm{ml}$ aliquots of serum sample were obtained by centrifugation ( $3500 \mathrm{rpm}$ for $10 \mathrm{~min}$ at $25-27^{\circ} \mathrm{C}$ ) and were store at $-20{ }^{\circ} \mathrm{C}$ until analysis. All the lipid measurements were performed on an automatic biochemical analyzer ILab Aries . ${ }^{\circledR}$

Statistical analysis was performed using Microsoft Excel v2016 and SPSS v21. Qualitative data presented as number and percentage while quantitative data presented as Mean $\pm \mathrm{sd}$. The student's t-test was used for quantitative variables and chi - square used for qualitative variables. Correlation coefficients and regression analysis used when appropriate. P-value of $<0.05$ considered as statistically significant.

\section{RESULTS}

In table 1, the average Weight gain of the pregnant in 2nd-trimester, was $3.7 \pm 0.65 \mathrm{~kg}$, while in $3 \mathrm{rd}$ trimester it was $4.68 \pm 0.57 \mathrm{~kg}$. During the $2 \mathrm{nd}-$ trimester assessment, 5 women developed maternal complications. In the 3rd-trimester assessment, 33 more women developed maternal complications as presented.

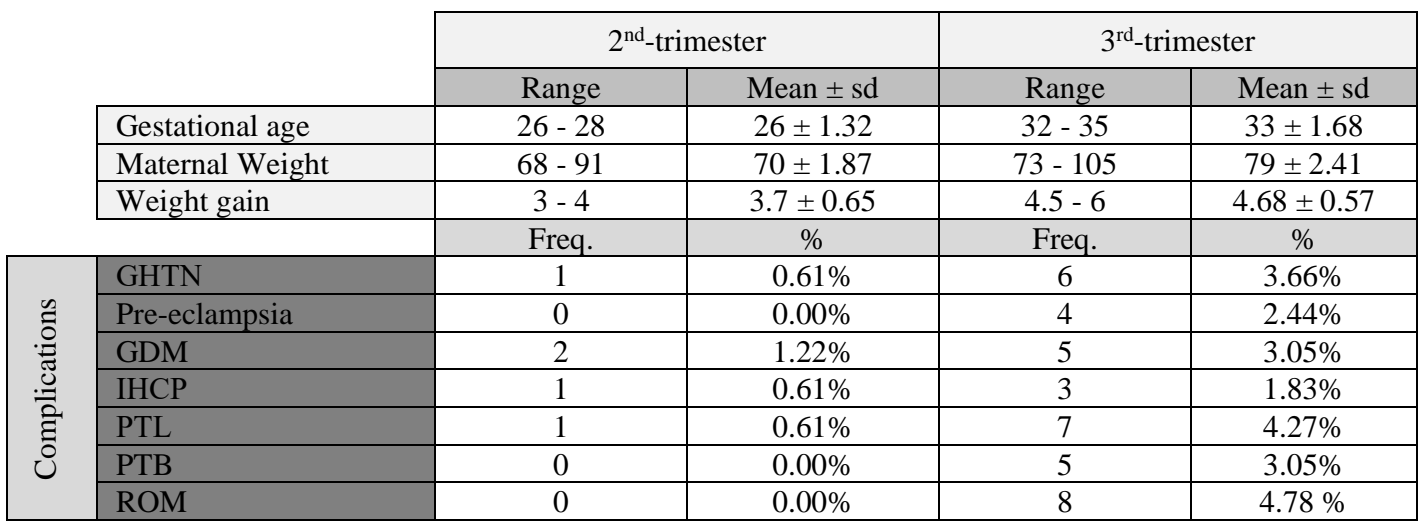

Table 1. Clinical data of the participants.

In Table 2, the average TC level during 3rd-trimester was highest in GHTN than in other complications. We observed that the level of HDL-C was decreased in patient $\mathrm{s}$ with IHCP. There are statistically significant difference between values of each lipid profile between 2nd and 3rd-trimester $\mathrm{v}$ occurrence of GHTN, GDM, IHCP, and PTL $(\mathrm{P}=0.00,0.00,0.02$, and 0.00 respectively)).

In Table 3, Multivariate analysis shows that every $\mathrm{mg} / \mathrm{dl}$ increase in 3rd-trimester TG concentration was associated with an increased risk for SGA (AOR= 1.12), $\mathrm{LGA}(\mathrm{AOR}=1.21)$, and macrosomia $(\mathrm{AOR}=$ 1.21). On contrary, every $\mathrm{mg} / \mathrm{dl}$ increase in $3 \mathrm{rd}$ - trimester TG concentration was associated with decreased risk for the Low Apgar score (AOR= $0.523)$.

Table 4 shows a significant association between the level of maternal TG and occurrence of SGA, LGA, Macrosomia Low Apgar score $(\mathrm{P}=0.046,0.025,0.024$ and 0.091 respectively).

In table 5, TG had a strong predictive power for GHNT/PE and SGA at a cut-off point of $309.21 \mathrm{mg} / \mathrm{dl}$. Also, the cut-off point of 291.45 and 254.32 for TC had higher sensitivity for predicting GDM and GHTN respectively. On the other side, the optimal cut-off 
points for $3^{\text {rd }}$-trimester HDL-C in identifying IHCP and SGA was $52.42 \mathrm{mg} / \mathrm{dl}$ and $88.2 \mathrm{mg} / \mathrm{dl}$ respectively. The optimal cut-off point $113.12 \mathrm{mg} / \mathrm{dl}$ for LDL-C has a sensitivity of $73.3 \%$ for predicting LGA.

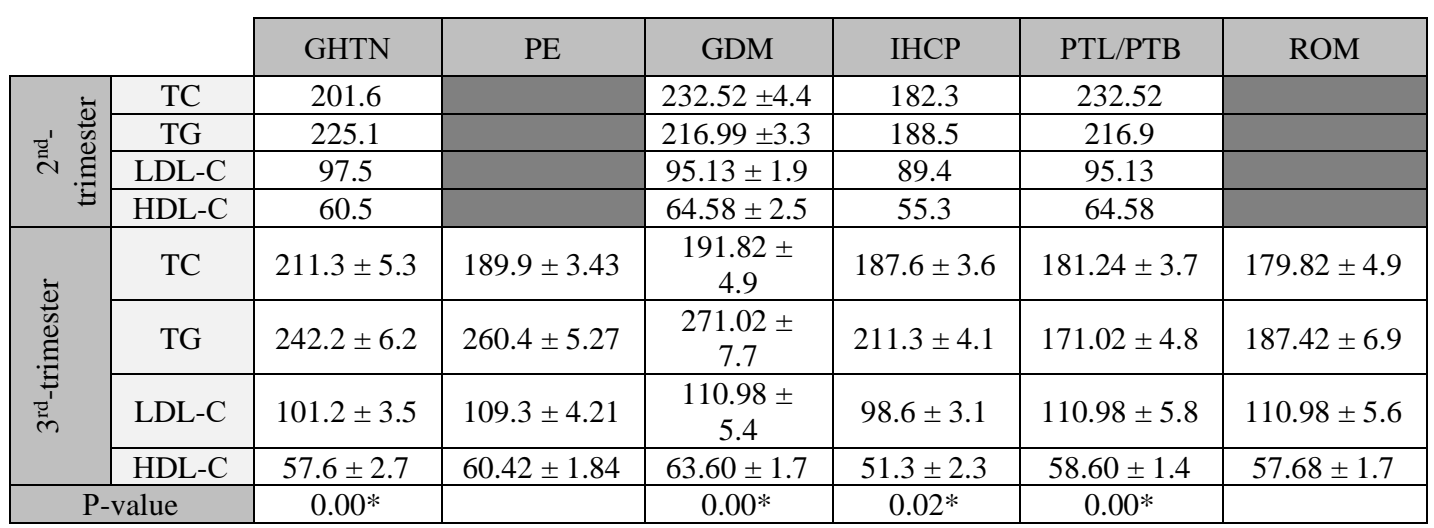

Table 2. Lipid profile parameters in complicated pregnancies.

\begin{tabular}{|c|c|c|c|c|c|c|c|}
\hline & \multicolumn{6}{|c|}{ Maternal complications } \\
\hline & & GDM & GHTN & $\mathrm{PE}$ & IHCP & PTL & ROM \\
\hline \multirow{3}{*}{$\mathrm{TC}$} & AOR & 0.84 & 1.17 & 0.99 & 1.20 & 1.37 & 0.52 \\
\hline & $\mathrm{CI}$ & $0.68-1.04$ & $1.04-1.61$ & $0.64-1.54$ & $0.97-1.49$ & $1.06-1.42$ & $0.13-0.58$ \\
\hline & P-value & 0.009 & 0.00 & 0001 & 0.096 & 0.142 & 0.131 \\
\hline \multirow{3}{*}{ TG } & $\mathrm{AOR}$ & 1.37 & 1.10 & 1.50 & 1.28 & 0.52 & 0.84 \\
\hline & CI & $1.181-.58$ & $0.89-1.52$ & $1.16-1.93$ & $1.09-1.51$ & $0.71-1.63$ & $0.67-1.48$ \\
\hline & P-value & 0.00 & 0.00 & 0.002 & 0.002 & 0.085 & 0.071 \\
\hline \multirow{3}{*}{ HDL-C } & AOR & 0.52 & 0.62 & 0.65 & 0.59 & 0.48 & 1.37 \\
\hline & CI & $0.38-1.72$ & $0.31-1.28$ & $0.33-1.31$ & $0.64-1.16$ & $1.27-1.89$ & $0.99-1.32$ \\
\hline & P-value & 0.00 & 0.00 & 0.228 & 0.003 & 0.061 & 0.058 \\
\hline \multirow{3}{*}{ LDL-C } & AOR & 1.27 & 1.22 & 0.48 & 0.56 & 1.37 & 1.37 \\
\hline & $\mathrm{CI}$ & $0.73-1.58$ & $0.57-1.61$ & $0.10-2.26$ & $0.25-1.22$ & $0.76-1.23$ & $0.68-1.04$ \\
\hline & P-value & 0.001 & 0.00 & 0.352 & 0.141 & 0.103 & 0.121 \\
\hline
\end{tabular}

Table 3. Associations between maternal 3rd-trimester lipid profile and maternal complications.

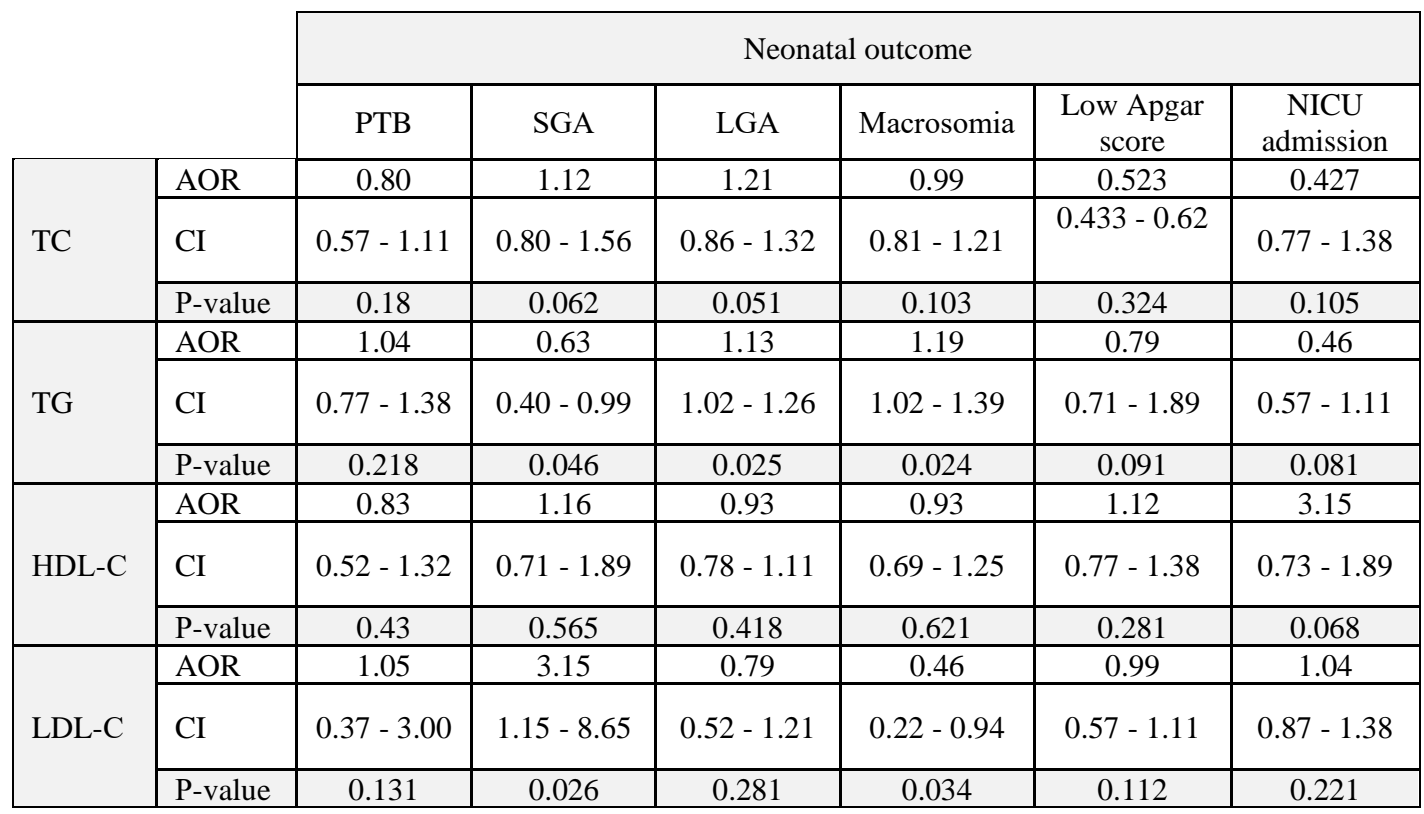

Table 4. Associations between maternal 3rd-trimester lipid profile and neonatal outcome. 


\begin{tabular}{|c|c|c|c|c|c|c|}
\hline \multirow{2}{*}{ Outcomes } & \multirow{2}{*}{$\begin{array}{l}\text { Cut-off point } \\
(\mathrm{mg} / \mathrm{dl})\end{array}$} & \multirow{2}{*}{ AUC } & \multicolumn{2}{|c|}{ Confidence interval $95 \%$} & \multirow{2}{*}{$\begin{array}{c}\text { Sensitivity } \\
(\%)\end{array}$} & \multirow{2}{*}{$\begin{array}{c}\text { Specificity } \\
(\%)\end{array}$} \\
\hline & & & Min & Max & & \\
\hline \multicolumn{7}{|l|}{ TC } \\
\hline GHNT/PE & 254.32 & 0.713 & 0.538 & 0.788 & 82.3 & 64.2 \\
\hline SGA & 164.23 & 0.684 & 0.557 & 0.715 & 78.4 & 68.3 \\
\hline \multicolumn{7}{|l|}{ TG } \\
\hline GDM & 242.85 & 0.708 & 0.572 & 0.742 & 62.1 & 47.8 \\
\hline GHNT/PE & 259.21 & 0.713 & 0.538 & 0.788 & 82.3 & 64.2 \\
\hline LGA & 261.21 & 0.713 & 0.538 & 0.788 & 82.3 & 64.2 \\
\hline \multicolumn{7}{|l|}{ HDL-C } \\
\hline GHNT/PE & 72.54 & 0.795 & 0.532 & 0.645 & 74.3 & 65.4 \\
\hline IHCP & 52.42 & 0.842 & 0.677 & 0.911 & 88.2 & 74.4 \\
\hline SGA & 60.45 & 0.647 & 0.531 & 0.798 & 62.1 & 73.1 \\
\hline \multicolumn{7}{|l|}{ LDL-C } \\
\hline GHNT/PE & 109.76 & 0.778 & 0.584 & 0.765 & 64.2 & 65.4 \\
\hline LGA & 113.12 & 0.737 & 0.517 & 0.684 & 73.3 & 54.3 \\
\hline
\end{tabular}

Table 5. Optimal cut-off points of maternal 3rd-trimester lipids for predicting pregnancy complications and perinatal outcomes.

\section{DISCUSSION}

During early pregnancy, there is an increase in body fat accumulation, associated with both hyperphagia and increased lipogenesis which is necessary as an energy store to fulfill maternal and fetal metabolic needs. ${ }^{6}$

TG rises disproportionately in comparison to other lipid parameters reaching 2 - 4 times pre-pregnancy levels by the $3^{\text {rd }}$-trimester, which fall precipitously to pre-pregnancy levels after delivery. ${ }^{7}$

In our study, the serum level of TC, TG and LDL-C increased with increasing gestational age and peaked before delivery, while serum level of HDL-C dropped a little in the $2^{\text {nd }}$-trimester with more decrease in the $3^{\text {rd }}$-trimester. Our results of our study are consistent with other studies. $8,9,10$

Many hypotheses were built to explain the state of dyslipidemia and changes in the lipids metabolism during pregnancy. The rise in plasma lipids influenced by the placental hormones especially in the $3^{\text {rd }}$ trimester. ${ }^{11}$ Estrogen induces hepatic biosynthesis of endogenous triglycerides, which is carried by VLDL, while progesterone oppose the action estrogens on lipoprotein metabolism. ${ }^{4}$

During pregnancy the hepatic lipase activity and reduced lipoprotein lipase activity increased resulting in increase in circulating TGs. ${ }^{12}$

In our study, we observe a significant difference in the mean values of the lipid profile between complicated and uncomplicated pregnancies. This makes it sense that a state of dyslipidemia may provoke the occurrence of maternal complications.

In our study, the serum level of maternal TC, TG and LDL-C, were increased in GHNT when compared to normotensive pregnancy, which is statistically significant $(\mathrm{P}=0.012,0.00,0.00$ respectively). Also, the level of HDL-C in women with GHNT was lower than normotensive women. We observe that the serum level of HDL was lower more in cases with $\mathrm{PE}(\mathrm{P}=$ $0.010)$.

Our results are in agreement with the study of Shen, ${ }^{14}$ White, ${ }^{15}$ Vani, ${ }^{16}$ Nayan, ${ }^{17}$ and Singh, ${ }^{18}$ et al.

Previous studies shown that plasma lipids rise significantly in preeclampsia above the levels of normal pregnancies. ${ }^{19}$ An association between the atherogenic lipid profile of gestation and the endothelial cell dysfunction during preeclampsia has been described. ${ }^{20}$

We observed every $\mathrm{mg} / \mathrm{dl}$ elevation in maternal $3^{\text {rd }}$ trimester TG concentration was associated with an increased risk of GDM, GHTN, preeclampsia, and IHPC. Also, every unit increase in HDL-C was associated with an increased risk of IHCP, GDM and GHNT. Besides, every unit increase in LDL-C reduced the risk of GDM and GHNT. Moreover, every unit increase in TC reduced the risk of GHTN. Our results were in line with the study of Jin et al. ${ }^{21}$

We observed that high levels of TGs during pregnancy were associated with an increased risk of GDM. During $2^{\text {nd }}$ and $3^{\text {rd }}$ trimesters maternal fuel adjustments occur which leads to the sparing of glucose for the fetus leading to GDM. Freinkel had described these changes as "accelerated starvation", and "facilitated anabolism". ${ }^{22}$

Women with GDM characterized by a predominant insulin sensitivity defect had significantly higher triglycerides, lower HDL-C, and higher NEFA when compared with women with normal glucose test. ${ }^{23}$

There was a significant association between the level of maternal TG and occurrence of SGA, LGA, 
Macrosomia and Low Apgar score. Also, there is a significant association between the level of maternal HDL-C and the occurrence of SGA and Macrosomia. There was no significant association between $3^{\text {rd }}$ trimester TC and LDL-C with the occurrence of neonatal complications (PTB, SGA, LGA, Macrosomia, Low Apgar score, NICU admission). Jin et al. ${ }^{20}$ observed that every unit elevation in $3^{\text {rd }}$ trimester TG concentration was associated with increased risk for LGA, macrosomia and decreased risk for SGA.

Several previous studies have investigated associations between maternal lipid levels during pregnancy and risk for preterm birth. ${ }^{24,25,26}$

Hyperlipidemia is considered as an instigator of inflammation and oxidative stress which are a risk factor for PTB. Previous studies indicated an increased risk of PTB associated with maternal dyslipidemia. ${ }^{27}$

\section{CONCLUSION}

Maternal dyslipidemia is a risk factor and associated with the development and occurrence of maternal complications during pregnancy and affects the neonatal outcome. Testing lipid profile during 2 nd and 3rd trimesters can early predict certain disorders associated with pregnancy.

\section{REFERENCES}

1. Ekhator, C. N., and . Ebomoyi M. I. Blood glucose and serum lipid profiles during pregnancy. African Journal of Diabetes Medicine.2012; 20(1).

2. Grimes, S. B. and Wild, R.. Effect of pregnancy on lipid metabolism and lipoprotein levels. In Endotext [Internet]. MDText. com, Inc., 2018.

3. Straughen, J. K., Trudeau S, and. Misra V. K. Changes in adipose tissue distribution during pregnancy in overweight and obese compared with normal weight women. Nutrition and diabetes 2013; 3(8):84.

4. Festus OO, Idonije OB, Eseigbe MA, et al. Comparative study of lipid profile of normal pregnant women in the different trimesters. Arch Appl Sci Res. 2011, 3 (3):528-32.

5. Schaefer-Graf UM, Meitzner K, Ortega-Senovilla $\mathrm{H}$, et al. Differences in the implications of maternal lipids on fetal metabolism and growth between gestational diabetes mellitus and control pregnancies. Diabetic medicine: a journal of the British Diabetic Association. 2011; 28(9): 1053-9.

6. Herrera E. Lipid metabolism in pregnancy and its consequences in the fetus and newborn. Endocrine 2002; 19(1): 43-55.

7. Hadden DR and McLaughlin C. Normal and abnormal maternal metabolism during pregnancy. Seminars in Fetal and Neonatal Medicine 2009; 14(2): 66-71.

8. Pusukuru R, Shenoi AS, et al. Evaluation of Lipid Profile in Second and Third Trimester of Pregnancy. Journal of clinical and diagnostic research: $2016 ; 10(3)$ : 12-6.
9. Farias DR, Poston L, Franco-Sena AB, et al. Maternal lipids and leptin concentrations are associated with large-for-gestational-age births: a prospective cohort study. Scientific Reports. 2017; 7(1): 1-15.

10. Vahratian A, Misra VK, Trudeau S, et al. Prepregnancy body mass index and gestational age-dependent changes in lipid levels during pregnancy. Obstetrics and Gynecology. 2010; 116(1): 107-13.

11. Setareh A, Mitra MG, Sedigheh B, et al. Maternal plasma lipid concentrations in first trimester of pregnancy and risk of severe pre-eclampsia. $\mathrm{PakJ}$ Med Sci. 2009; 25(4): 563-7.

12. Aziz R and Mahboob T. Pre-eclampsia and lipid profile. Pakistan Journal of Medical Sciences 2007; 23(5): 751.

13. Vrijkotte TG, Krukziener N, Hutten BA, et al. Maternal lipid profile during early pregnancy and pregnancy complications and outcomes: the ABCD study. The Journal of Clinical Endocrinology and Metabolism. 2012; 97(11): 3917-25.

14. Shen H, Liu X, Chen Y, et al. Associations of lipid levels during gestation with hypertensive disorders of pregnancy and gestational diabetes mellitus: a prospective longitudinal cohort study. BMJ Open. 2016; 6(12): 13509.

15. White, I. Putu FI, John JE Wantania, et al. Differences in Serum Lipid Profile Value in Pregnancy and Postpartum on Severe Preeclampsia. Indonesian Journal of Obstetrics and Gynecology. 2019: 15-20.

16. Vani I, Gayathri A, Nagamani T., et al. Lipid Profile Parameters in Normal and Preeclampsia Complicating Pregnancies- A Prospective Observational Study. The American Journal of Science and Medical Researc. 2015;1(1):61-6.

17. Nayan S, Meena ML, Hooja N, et al. A study of comparison of serum lipid profile of women with pregnancy-induced hypertension and normal pregnancy. Sch. Acad. J. Biosci. 2014; 2(11): 8346.

18. Singh U, Yadav S, Mehrotra S, et al. Serum lipid profile in early pregnancy as a predictor of preeclampsia. International Journal of Medical Research and Review. 2013, 1(2):56-62.

19. Bodnar LM, Ness RB, Harger GF., et al. Inflammation and triglycerides partially mediate the effect of prepregnancy body mass index on the risk of preeclampsia. American journal of epidemiology 2005, 162(12):1198-206.

20. Belo L, Caslake M, Gaffney D, et al. Changes in LDL size and HDL concentration in normal and preeclamptic pregnancies. Atherosclerosis. 2002, 162.(2): 425-32.

21. Jin WY, Lin SL, Hou RL, et al. Associations between maternal lipid profile and pregnancy complications and perinatal outcomes: a population-based study from China. $B M C$ pregnancy and childbirth. 2016; 16(1): 60.

22. Freinkel, N. Effects of the conceptus on maternal metabolism during pregnancy. On the nature and treatment of diabetes. 1965: 679-91.

23. Layton J, Powe C, Allard C, et al. Maternal lipid profile differs by gestational diabetes physiologic subtype. Metabolism. 2019; 91: 39 - 42. 
24. Smith CJ, Baer RJ, Oltman SP., et al. Maternal dyslipidemia and risk for preterm birth. PloS one 2018; 13(12): 209579.

25. Moayeri M, Heida KY, Franx A, et al. Maternal lipid profile and the relation with spontaneous preterm delivery: a systematic review. Archives of gynecology and obstetrics. 2017; 295(2): $313-23$

26. Jiang S, Jiang J, Xu $\mathrm{H}$, et al. Maternal dyslipidemia during pregnancy may increase the risk of preterm birth: A meta-analysis. Taiwanese Journal of Obstetrics and Gynecology. 2017; 56(1): 9 - 15.

27. Hendler I, Goldenberg RL, Mercer BM, et al. The Preterm Prediction Study: association between maternal body mass index and spontaneous and indicated preterm birth. American journal of obstetrics and gynecology. 2005; 192(3): 882 - 6. 